\title{
EXPERIMENTAL STUDY REGARDING THE EFFECTS OF PULSED SHORT WAVES ON NERVOUS TISSUE
}

\author{
DOGARU Gabriela ${ }^{1,2}$, CRACIUN Constantin ${ }^{3}$
}

\author{
${ }^{1}$ Clinical Rehabilitation Hospital Cluj-Napoca \\ 2"Iuliu Hațieganu" University of Medicine and Pharmacy Cluj-Napoca \\ 3،"Babeş-Bolyai" University Cluj-Napoca, Center of Electron Microscopy
}

\begin{abstract}
Pulsed short waves are part of high frequency therapy and have through their biological effects, the good therapeutic results and the lack of side effects many indications in various disorders: rheumatologic, traumatologic, burns, neurological (nerve tissue regeneration, ischemic vascular accidents), vascular disorders. The experimental study consisted of the exposure of laboratory animals to the action of pulsed short waves in different doses and the monitoring of changes in the brain cellular and subcellular structure. The study included 35 Wistar rats, assigned to four groups, three groups of 10 animals and the control group of five non-irradiated animals. Group I was exposed to a dose of $1 / 80$ cycles/sec, group II to $4 / 400 \mathrm{cycles} / \mathrm{sec}$, and group III to $6 / 600 \mathrm{cycles} / \mathrm{sec}$, for 10 minutes/day, for 15 days. After the rats were sacrificed, nervous tissue fragments were collected. These were adequately processed for their examination by optical and electron microscopy. At structural level, in the control group and the groups exposed to the dose of $1 / 80 \mathrm{cycles} / \mathrm{sec}$, the neurons were normally structured. In group II, a slight increase of brain metabolic activity was seen, in group III there was a significant increase of brain metabolism, with better myelinated white matter axons, which suggests a better protection for the rapid conduction of nerve impulses to effectors. In group II, electron microscopy revealed similar aspects to those of the control group. A high ribosome density was found in the neuroplasm, which means an intense protein synthesis process. In group III, there was an intense protein synthesis activity, and a strong axon myelination in the white matter, resulting in a better protection for the transmission of nerve impulses. Conclusions: pulsed short waves caused brain changes depending on the dose. The analysis of electron microscopic images showed that irradiation at a dose of 6/600 cycles/sec induced an intense protein synthesis activity and a better axon myelination, which conferred a better protection for the transmission of impulses, more obvious than in the group irradiated at a dose of 4/400 cycles/sec.
\end{abstract}

Keywords: pulsed short waves, Diapulse, nervous tissue, experimental research 


\section{Introduction}

Pulsed short waves are short electromagnetic waves emitted as intermittent trains with a fixed duration, separated by free intervals with a variable duration [1]. The Diapulse device provides high frequency currents of $27.12 \mathrm{MHz}$, with a wave length of $11.6 \mathrm{~m}$, providing rectangular pulse trains with a duration of 65 microseconds [2].

Experimental research as well as the multitude of therapeutic effects observed in clinical studies suggest complex action mechanisms up to the infrastructure level, with deep implications in cellular metabolism. If the favorable effects of short continuous waves are mostly due to the endotissular production of heat (endothermy) [2], in the case of pulsed short waves, the action mechanism is more complex; these are athermal, acting on energy metabolism [1].

There are many experimental studies on laboratory animals, cell and tissue cultures that have been aimed at evidencing the biological effects and elucidating the action mechanisms of pulsed short waves. The effects of pulsed short waves on the regeneration and remyelination of peripheral nerves after lesions, the sectioning of the common peroneal nerves in male rats $[3,4]$, of the sciatic nerve in cats [9] have been experimentally studied.

Human and animal studies indicate the fact that pulsed short wave treatment shortly after spinal cord injuries protects the neurons, promotes regeneration and minimizes the lost function.

Dr. H. Crock (London) emphasized the fact that the blood flow is the main factor to be approached after spinal cord injuries.
Considering the ability of pulsed short waves to increase blood flow, studies indicate favorable effects on the healing of spinal cord injuries [10, 11].

Dr. Wise Young (New York, 1984) showed by laboratory studies on cats that the application of Diapulse therapy on the spinal cord shortly after injury reduces calcium accumulation in the proximity of the affected area. This is associated with a better recovery of spinal cord motor and sensory functions [12]. It was concluded that pulsed short waves can exert a regenerative and neuroprotective influence if administered shortly after spinal cord injury.

An experimental study consisted of the exposure of laboratory animals, i.e. white Wistar rats, to the action of pulsed short waves in different doses (1/80, 4/400, $6 / 600 \mathrm{impulses} / \mathrm{sec}$.) for $10 \mathrm{~min} / \mathrm{day}$, for 15 days, and the monitoring of cellular and subcellular structure changes occurring in various organs and tissues. It was found that the most sensitive organs to the action of these waves were the liver, spleen, adrenal gland, brain, their alterations depending on the dose used [13]. In extensive clinical studies, Dr. M. Sambasivan (India, 1993) showed that Diapulse therapy reduced cerebral edema after cranial trauma [14].

An experimental study on Wistar and Brown Norway rats demonstrated the capacity of the pulsed electromagnetic field to stimulate the regeneration of the peripheral nervous system, of the sciatic nerve, respectively. Treatment was administered for four weeks. The results were evaluated by quantitative morphometric analysis, demonstrating a statistically significant increase of regeneration indices in the stimulated group compared to the control group. 
An increase of isocitrate dehydrogenase and acetyl cholinesterase activity at the motor plate was seen. This study might lead to the search of new alternatives in the stimulation of axon regeneration processes and possible clinical applications [7]. There are currently no clinical studies regarding the effects of pulsed short waves in the regeneration of the peripheral nervous system. Pulsed short waves are part of high frequency therapy and due to their biological influence, effective therapeutic results and absence of side effects, they have many indications in various diseases: rheumatologic diseases, trauma, burns, neurological disorders (regeneration of nerve tissue, stroke), vascular diseases.

\section{Material and method}

The experimental study consisted of the exposure of laboratory animals to the action of pulsed short waves in different doses and the monitoring of cellular and subcellular structure changes in nervous tissue.

Experimental animals. White female Wistar rats with a weight of 180$280 \mathrm{~g}$ were used.

Equipment. The Diapulse device was used.

Experimental model. 35 animals were assigned to 4 groups. Group I included 10 experimental animals exposed to radiation in a dose of 1/80 impulses/sec for 10 $\mathrm{min} /$ day, group II consisted of 10 animals exposed to a dose of $4 / 400$ impulses/sec for $10 \mathrm{~min} /$ day, group III was formed by 10 animals exposed to a dose of $6 / 600$ impulses/sec for $10 \mathrm{~min} /$ day, and the control group included 5 unexposed animals, which were kept under the same living conditions as the animals of the three irradiated groups.
The duration of the experiment was 15 days. The entire body of the animals was exposed to the pulsed short waves.

There were no food or liquid restrictions during the experiment.

All procedures performed on the animals were in accordance with the ethical norms required by the EU regarding the raising and sacrificing of experimental animals. Before starting the experiment, the approval of the Ethics Board no. 39/23.04.2009 was obtained.

Right after the animals were sacrificed, nervous tissue and brain fragments were collected and processed according to the methods and techniques for optical and electron microscopy examination.

From the blocks obtained and modeled adequately, semithin sections $500 \mathrm{~nm}$ thick were cut, for optical microscopy studies. The sections were examined with an Olympus BX 51 microscope, and images were captured with a CCD Media Cybernetics camera, using the Image Pro Plus software.

Subsequently, from the selected areas of the same blocks, ultrathin sections 40-60 $\mathrm{nm}$ thick were cut, for electron microscopy studies. The sections were cut with a Leica UC 6 ultramicrotome using DDK diamond knives, they were collected on electrolytic grids, and were double contrasted with uranyl acetate and lead citrate, to be finally examined with a Jeol JEM 1010 transmission electron microscope (TEM). Images were captured with a Megaview III camera and stored in the database using the Soft Imaging Analysis software [15, 16, $17,18]$. 


\section{Results and discussion}

\section{Structural changes}

In the control group and group I (1/80 impulses/sec), the presence of many normally structured neurons was evidenced in the grey matter. The neuronal body of each neuron had in its center a spherical euchromatic nucleus, with a single nucleolus. Each nucleus was surrounded by the cytoplasm (neuroplasm), which sent an axonal projection. The neurons were surrounded by fragmentary portions of multitudes of neuronal projections (axons, dendrites).

In some grey matter areas, aged neurons were seen, which were in the process of degeneration that naturally occurs during the course of life of each individual. In the white matter, a multitude of normally myelinated axons were evidenced.

In group II (4/400 impulses/sec), a slight increase of brain metabolism activity was comparatively seen, which was evidenced by the presence of a higher number of normally structured neurons. Like in the case of the control group, aged neurons in the process of degeneration were also found.

In the white matter, like in the control group, the multitude of axons had a normally myelinated sheath (Fig. 1).

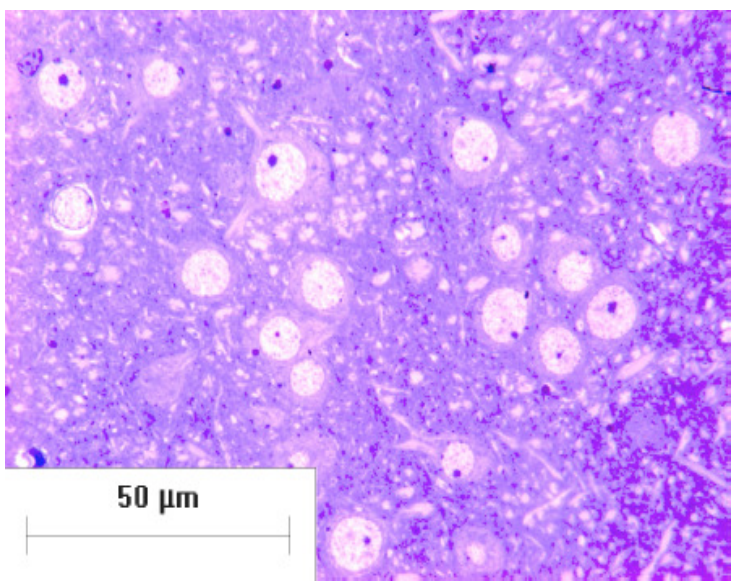

Fig. 1 - Brain. Group II. Normally structured neurons. $400 \mathrm{x}$.
At a 6/600 cycles/sec dose, the multitude of normally structured neurons indicated a significant increase in brain metabolism compared to the previous groups. The neurons were slightly more numerous in relation to the surface area unit per brain volume (Fig. 2). The number of aged neurons in the process of degeneration was smaller than in the previous groups, and the blood capillaries were normally structured. In the white matter, the multitude of axons had good myelination, suggesting a better protection for the rapidity of conduction of nervous impulses to the effectors (Fig. 3).

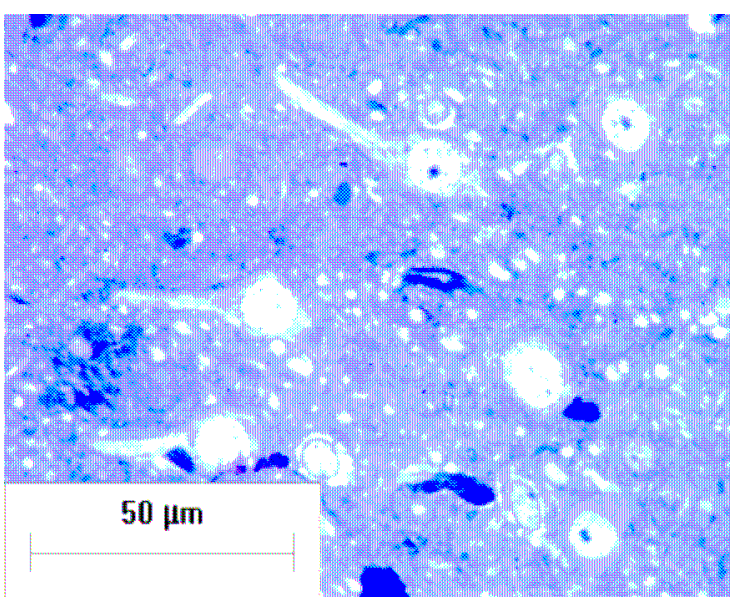

Fig. 2 - Brain. Group III. Normally structured neurons. $100 \mathrm{x}$

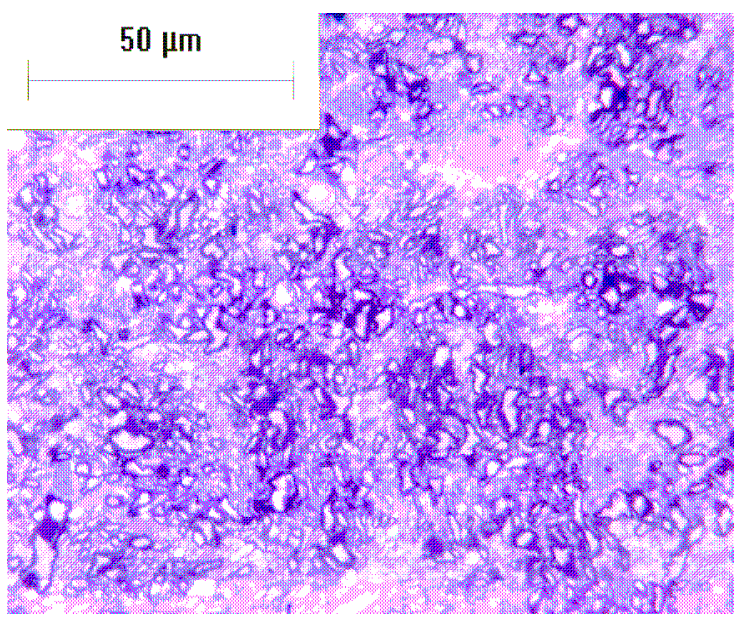

Fig. 3 - Brain. Group III. Axons with good myelination. $100 \mathrm{x}$ 


\section{Ultrastructural changes}

In the control group and group I, electron microscopic images showed many normally structured neurons, in which each neuronal cell had a spherical euchromatic nucleus occupying most of the cell volume, with a single nucleolus.

In the cytoplasm (neuroplasm), narrow GER profiles (Nissl bodies), mitochondria, lysosomes, Golgi complex and many free and polysomal ribosomes were visible.

From each neuron, an axon started, in the axoplasm of which many neurotubules arranged parallel along the axonal projection, mitochondria and rare narrow GER profiles were seen.

From place to place, neurons at different degeneration stages were evidenced. The nuclei of these neurons lost their spherical shape, becoming hyperchromatic, dense, and in the neuroplasm, GER profiles and mitochondria were dilated and the cytoplasmic matrix was dense.

In the white matter, axons were normally myelinated and blood vessels had a normal structure (Fig. 4).

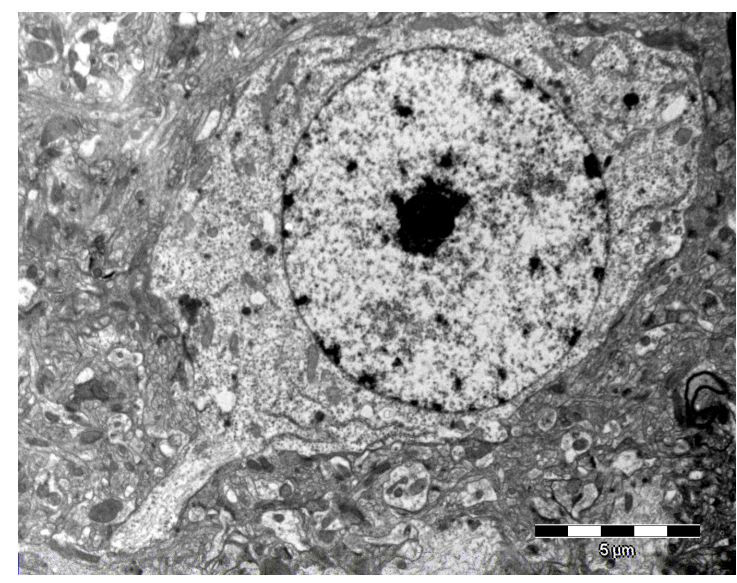

Fig. 4 - Brain. Control group. Neurons with a normal structure.
The ultrastructural aspects of the brain of rats irradiated at a 4/400 cycles/sec dose were similar to those of the control group. In the neuroplasm, several GER profiles were identified, as well as a higher density of ribosomes and polysomes, signifying the presence of an intense protein synthesis process (Figs. 5-6).

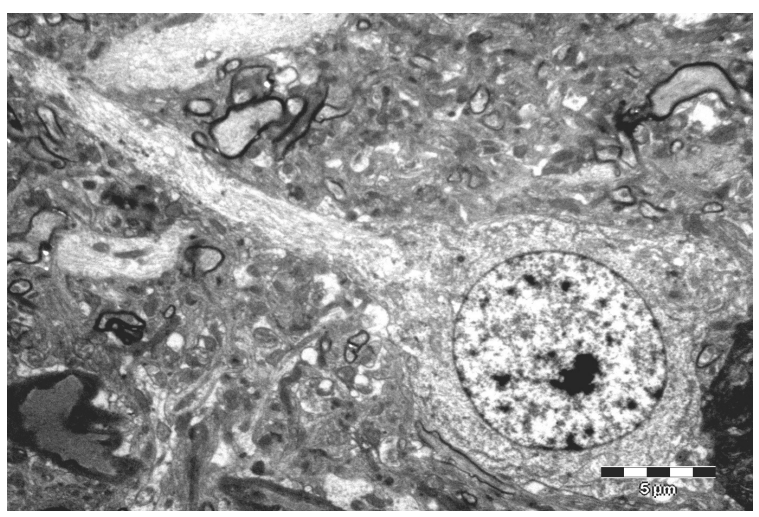

Fig. 5 - Brain. Group II. Neuroplasm with GER profiles.

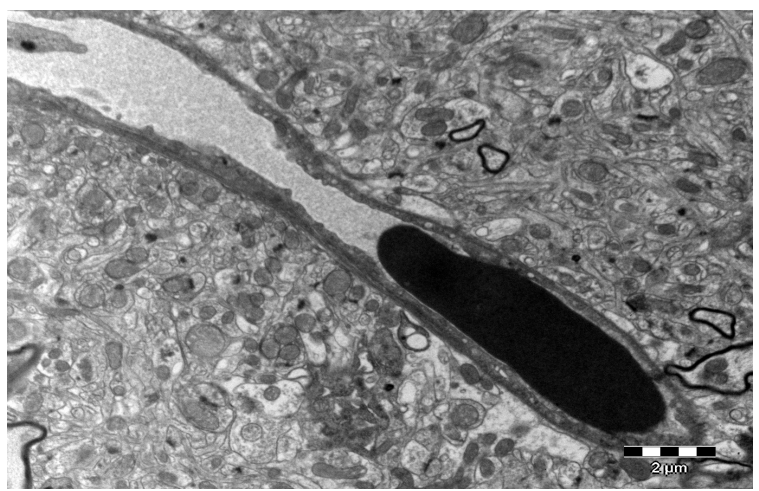

Fig. 6 - Brain. Group II. Normally structured blood vessel.

In the group irradiated at a $6 / 600$ cycles/sec dose, a higher density of neurons found in a high metabolic state was seen. In the cytoplasm, GER was more abundant, suggesting a high protein synthesis activity, more obvious than in the group irradiated at a 4/400 cycles/sec dose (Fig.7). In the axon, the neurotubules ran parallel to its long axis, suggesting the adequate direction and conduction of the nervous impulse (Fig. 8). 
In the white matter, there was a strong myelination of the axons, so that in some axons the myelin layer appeared to be thicker, conferring a better protection for the transmission of nervous impulses (Fig. 9). The blood vessels, both blood capillaries and venules, were normally structured.

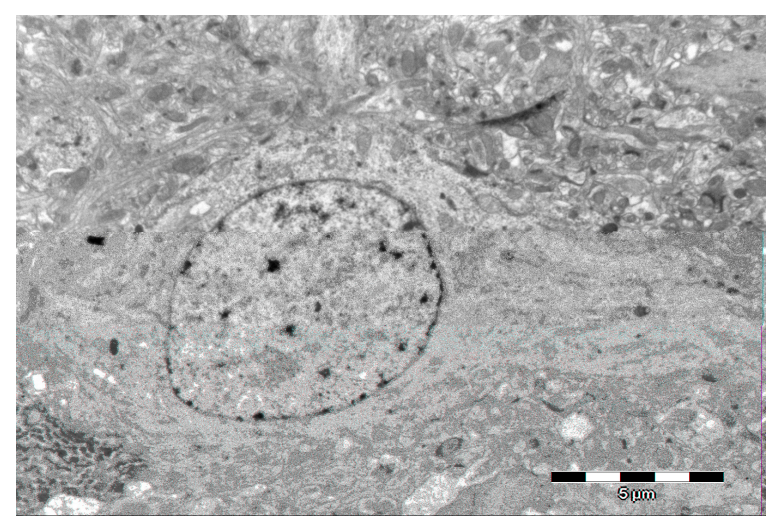

Fig. 7 - Brain. Group III. Cytoplasm with abundant GER.

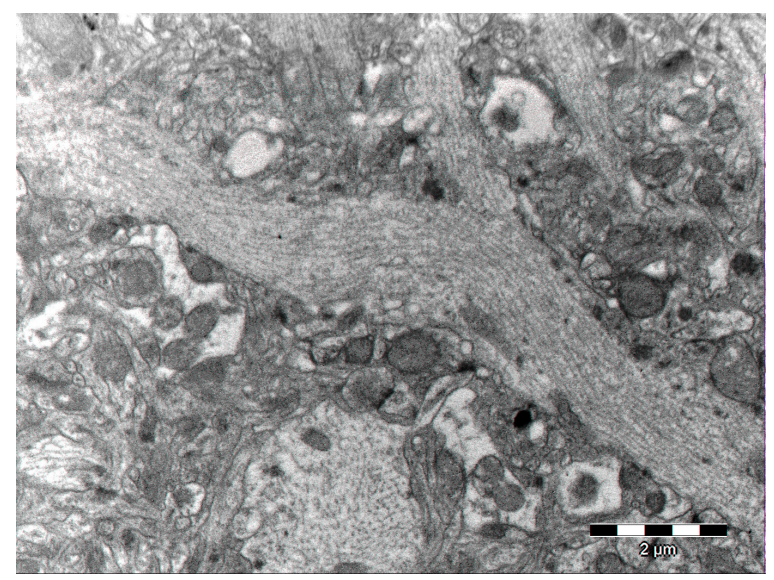

Fig. 8 - Brain. Group III. Axon with parallel neurotubules.

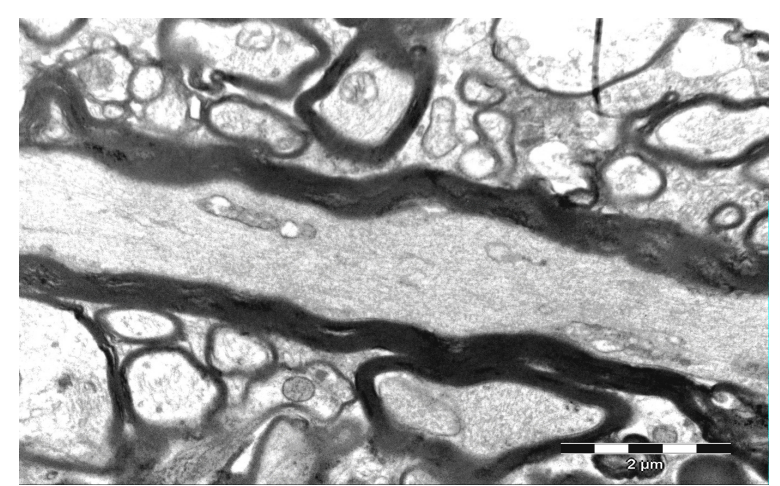

Fig. 9 - Brain. Group III. White matter, strong myelination of axons.

\section{Conclusions}

Pulsed short waves induced changes in the brain, depending on the dose. The analysis of electron microscopic images showed that exposure to a dose of $6 / 600$ cycles/sec induced intense protein synthesis activity and better axonal myelination, which ensured a better protection for the transmission of nerve impulses, more obvious than in the group exposed to the dose of 4/400 cycles/sec. 


\section{Bibliography}

1. Pop L. Cercetări clinico-experimentale asupra efectelor biologice şi terapeutice ale undelor scurte pulsatile (Diapulse). Teză de doctorat. U.M.F. „Iuliu Hațieganu”. Cluj-Napoca, 1985.

2. Rădulescu A. Electroterapie. Editura Medicală Bucureşti, 2004 .

3. Raji ARM., Bowden RE. Effects of high-peak pulsed electromagnetic field on the degeneration and regeneration of the common peroneal nerve in rats. $\mathrm{J}$ Bone Joint Surg Br. 1983; 65 (4): 478-92.

4. Raji ARM. An experimental study of the effects of pulsed electromagnetic field (Diapulse) on nerve repair. J Hand Surg Br. 1984; 9 (2): 105-112.

5. Sisken BF, Jacob JM, Walker JL. Acute treatment with pulsed electromagnetic fields and its effects on fast axonal transport in normal and regenerating nerve. J Neurosei Res. 1995; 42 (5): 692-699.

6. Sisken BF, Orgel M, Walker JL. Prospects on clinical applications of electrical stimulation for nerve regeneration. J Cell Biochem. 1993; 51 (4): 404-409.

7. Pedro JA, Coballer P, Dominiquez J, Collia F, Blanco J, Salvadoro M. Pulsed electromagnetic fields induce peripheral nerve regeneration and endplate enzymatic changes. Bioelectromagnetics 2005; 26: 20-27.

8. Wilson DH . The effects of pulsed electromagnetic energy on peripheral nerve regeneration. Annals of the New York Academy of Sciences 1974; vol. 238: 575580 .

9. Wilson DH, Jagadeesh P. Experimental regeneration in peripheral nerves and the spinal cord in laboratory animals exposed to a pulsed electromagnetic field. Paraplegia 1976; 14: 12-20.
10. Ellis W. Pulsed subcutaneous electrical stimulation in spinal cord injury. Bioelectromagnetics 1987; 8 (2): 159-164. 11. Kiwerski J, Chrostowska T, Weiss M. Clinical trials pf the application of pulsating electromagnetic energy (Diapulse) in the treatment of spinal cord lesions. Chir. Narz. Ortop. Pot .1980; 45 (3): 273-277.

12. Young W. Pulsed electromagnetic fields alter calcium in spinal cord injury. The society of neurological surgeons, 1984.

13. Dogaru BG, Crăciun C, Cătoi C, Toader S, Pop L. Histological changes in organs and tissues of rats undergoing pulsed short wave treatment. Annals of RSCB 2009; XIV (2) : 73-79.

14. Sambasivan M. Pulsed electromagnetic field in management of head injuries. Neurology India 1993; 41: 56-59.

15. Crăciun C, Horobin R. A tissue processing schedule for parallel light and electron microscopy, Proc. Roy. Microsc. 1989 ; 24 (4) 223-224 .

16. Hayat MA. Principles and techniques electron microscopy. Biological Appl. Fourth Ed., Ed. Cambridge Univ. Press., 2000.

17. Kay D. Techniques for electron microscopy. Second Ed., Blackwell Sci. Publ. Oxford, 1967.

18. Kuo J. Electron Microscopy. Methods and Protocols. Second Edition. Humana Press, 2007. 